\title{
BMJ Open Catheter to vein ratio and risk of peripherally inserted central catheter (PICC)-associated thrombosis according to diagnostic group: a retrospective cohort study
}

\author{
Rebecca Sharp (10 , ${ }^{1}$ Peter Carr, ${ }^{2,3}$ Jessie Childs, ${ }^{4}$ Andrew Scullion, ${ }^{5}$ Mark Young, ${ }^{6}$ \\ Tanya Flynn, ${ }^{7}$ Carolyn Kirker, ${ }^{8}$ Gavin Jackson, ${ }^{9}$ Adrian Esterman ${ }^{1}$
}

To cite: Sharp R, Carr P, Childs J, et al. Catheter to vein ratio and risk of peripherally inserted central catheter (PICC)-associated thrombosis according to diagnostic group: a retrospective cohort study. BMJ Open 2021;11:e045895. doi:10.1136/ bmjopen-2020-045895

- Prepublication history for this paper is available online. To view these files, please visit the journal online (http://dx.doi. org/10.1136/bmjopen-2020045895).

Received 18 0ctober 2020 Accepted 23 June 2021

Check for updates

(c) Author(s) (or their employer(s)) 2021. Re-use permitted under CC BY-NC. No commercial re-use. See rights and permissions. Published by BMJ.

For numbered affiliations see end of article.

Correspondence to

Dr Rebecca Sharp;

Rebecca.Sharp@unisa.edu.au

\section{ABSTRACT}

Objectives Determine the effect of the catheter to vein ratio (CVR) on rates of symptomatic thrombosis in individuals with a peripherally inserted central catheter (PICC) and identify the optimal CVR cut-off point according to diagnostic group.

Design Retrospective cohort study.

Setting 4 tertiary hospitals in Australia and New Zealand. Participants Adults who had undergone PICC insertion. Primary outcome measure Symptomatic thrombus of the limb in which the PICC was inserted.

Results 2438 PICC insertions were included with 39 cases of thrombosis $(1.6 \% ; 95 \% \mathrm{Cl} 1.14 \%$ to $2.19 \%)$. Receiver operator characteristic analysis was unable to be performed to determine the optimal CVR overall or according to diagnosis. The association between risk of thrombosis and CVR cut-offs commonly used in clinical practice were analysed. A $45 \%$ cut-off ( $\leq 45 \%$ versus $\geq 46 \%$ ) was predictive of thrombosis, with those with a higher ratio having more than twice the risk (relative risk 2.30; $95 \% \mathrm{Cl} 1.202$ to $4.383 ; p=0.01$ ). This pattern continued when only those with malignancy were included in the analysis, those with cancer had twice the risk of thrombosis with a CVR greater than $45 \%$. Whereas the $33 \%$ CVR cut-off was not associated with statistically significant results overall or in those with malignancy. Neither the $33 \%$ or $45 \%$ CVR cut-off produced statistically significant results in those with infection or other nonmalignant conditions.

Conclusions Adherence to CVR cut-offs are an important component of PICC insertion clinical decision making to reduce the risk of thrombosis. These results suggest that in individuals with cancer, the use of a CVR $\leq 45 \%$ should be considered to minimise risk of thrombosis. Further research is needed to determine the risk of thrombosis according to malignancy type and the optimal CVR for those with a non-malignant diagnosis.

\section{INTRODUCTION}

Peripherally inserted central catheter (PICC)associated thrombosis is often uncomfortable, may result in loss of intravenous access

\section{Strengths and limitations of this study}

Large, multisite study with 2438 peripherally inserted central catheters (PICCs).

- First study to analyse risk of thrombosis associated with the $33 \%$ and $45 \%$ catheter to vein ratio (CVR) cut-off recommendations commonly used in clinical practice for PICC insertion.

- Analysed risk of thrombosis associated with CVRs according to diagnostic group.

- Unable to perform planned analysis (receiver operator characteristic analysis) to determine the optimal CVR to prevent thrombosis in individuals with a PICC.

- The use of a tapered PICC impacted the accuracy of the PICC diameter and hence CVR for those participants that had the tapered portion inserted.

for treatment and damage to the vasculature limiting further PICC insertions. In some cases, PICC associated thrombosis precipitates pulmonary embolism and post-thrombotic syndrome. ${ }^{12}$ Approximately $2 \%$ of individuals receiving antimicrobials as part of outpatient parenteral therapy develop thrombosis. ${ }^{3} 4$ While those receiving cancer treatment suffer much higher rates, with $4 \%-6 \%$ of consumers with a haematological malignancy and $2 \%-5 \%$ of those with a solid tumour developing PICC associated thrombosis. ${ }^{35-7}$

This adverse event can be explained using mechanisms related to Virchow's triad (stasis, endothelial damage and hypercoagulable state of the patient). PICCs may have a large impact on the interruption of blood flow (stasis). In a mechanical model, Nifong and McDevitt ${ }^{8}$ demonstrated that blood flow was dependant on the size of the catheter and cylinder (or vein) size and PICCs commonly used in clinical practice may impede blood flow up to $80 \%{ }^{8}$ 
PICC insertion decisions such as the use of an appropriate catheter to vein ratio (CVR) affect PICC-associated thrombosis rates. ${ }^{3}$ Contemporary insertion approaches include measurement of the target vein diameter using ultrasound and limiting the CVR to reduce the risk of thrombosis. ${ }^{9}$ Different CVR cut-offs are used in clinical practice, many sites use a 33\% CVR limit, that is only onethird of the vein should be occupied by the catheter. ${ }^{70-13}$ Other sites use a $45 \%$ CVR limit as advocated by the Infusion Therapy Standards of Practice (Infusion Nurses Society 2021) ${ }^{14}$ However, there is a lack of research investigating safe CVRs to use for PICC insertion. Previous research in an adult population that used receiver operator characteristic (ROC) analysis found that a $45 \%$ CVR was the optimal cut-off to reduce the risk of thrombus. ${ }^{15}$ Participants with a CVR of more than $45 \%$ were 13 times more likely to suffer from thrombosis. Yet these findings were based on just four cases and all participants with this adverse event had a haematological malignancy.

Most of the research investigating thrombosis rates associated with CVR cut-offs focus on individuals with cancer. ${ }^{571113}$ This is problematic as many consumers with an infection (without an underlying malignancy diagnosis) receive a PICC for antimicrobial treatment and it is unknown whether the CVR cut-off may differ according to diagnosis. There is a need to investigate the association between the CVR and PICC-associated thrombosis in a larger sample and to determine a safe CVR cut-off in individuals with both malignant and non-malignant health conditions. This study aimed to determine the effect of the CVR on rates of symptomatic thrombosis in individuals with a PICC, identify the optimal CVR cut-off point and determine if the CVR cut-off is the same for those with malignant and non-malignant disease.

\section{METHOD}

This was a retrospective cohort study set at hospitals in Australia (Calvary Mater Hospital, Newcastle, St Vincent's Hospital, Sydney and St George Hospital, Sydney) and New Zealand (Capital \& Coast District Health Board, Wellington). Clinicians from PICC services at each site used an existing PICC database and hospital information systems to populate a standardised spreadsheet. Data regarding PICC insertion from 2015 to 2018 was included.

\section{Inclusion criteria}

Adults who had undergone PICC insertion that terminated in the superior vena cava/right atrium junction.

\section{Exclusion criteria}

Cases where diagnosis, PICC size (Fr), external length and vein diameter measurement were missing.

Participants were allowed in the study more than once. PICCs were inserted as per usual clinical practice at each site. The anteroposterior diameter of the relevant vein (basilic, brachial or cephalic) was measured using ultrasound at the insertion point. No tourniquet

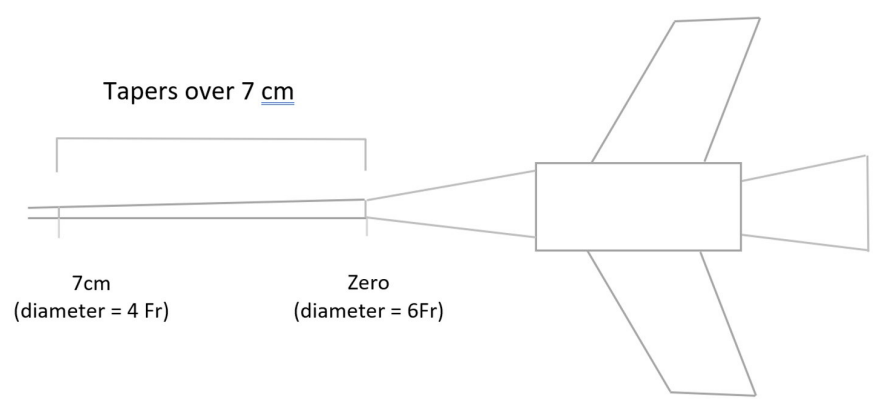

Figure 1 A 4Fr reverse taper PICC (image author's own). PICC, peripherally inserted central catheter.

was used during the measurement process to reflect the natural vein diameter. Veins were measured using a linear transducer angled at 90 degrees to the vein and from hypoechoic inner wall to inner wall of the vein excluding the echogenic rim of the vein. The measurement was conducted using inbuilt callipers in a Site $\sim$ Rite 8 Ultrasound System (C. R. Bard, Salt Lake City, Utah, USA) at Australian sites and a Sonosite micromax and SII at the New Zealand site (SonoSite, Bothell, Washington, USA).

A polyurethane, reverse taper PICC design was used by all sites (figure 1).

This catheter increases in diameter towards the hub (tapers $2 \mathrm{Fr}$ over $7 \mathrm{~cm})$. So that a $4 \mathrm{Fr}$ PICC is $4 \mathrm{Fr}(1.33 \mathrm{~mm})$ at $7 \mathrm{~cm}$ and $6 \mathrm{Fr}(2 \mathrm{~mm})$ at zero (near the hub). This is an increase in $0.67 \mathrm{~mm}$ over $7 \mathrm{~cm}$ towards the hub or $0.10 \mathrm{~mm}$ per $\mathrm{cm}$. For those participants with an external length $\leq 6 \mathrm{~cm}$, the external length (measured from insertion site to zero at sites) was used to determine the additional taper diameter for those PICCs (table 1). This measurement was added to the diameter of the PICC (Fr) as stated in the manufacturer information (outer diameter). For example, if a participant had a 4Fr PICC $(1.33 \mathrm{~mm})$ with an external length of $3 \mathrm{~cm}$, the additional taper diameter would be $0.4 \mathrm{~mm}$ and the overall PICC diameter would be $1.733 \mathrm{~mm}$. For participants with an external length $\geq 7 \mathrm{~cm}$ (tapered part of the PICC not inserted), manufacturer information was used to determine the PICC diameter.

The participant medical record number was used to access hospital information systems for sonography reports performed on the same upper extremity as the site of PICC insertion. De-identified reports were copied by clinicians at each site and these reports were reviewed

\begin{tabular}{|c|c|}
\hline External length (cm) & Additional taper diameter $(\mathrm{mm})$ \\
\hline 0 & 0.7 \\
\hline 1 & 0.6 \\
\hline 2 & 0.5 \\
\hline 3 & 0.4 \\
\hline 4 & 0.3 \\
\hline 5 & 0.2 \\
\hline 6 & 0.1 \\
\hline
\end{tabular}


by two members of the project team at the University of South Australia (one an accredited medical Sonographer) to determine cases of thrombus.

\section{Patient and public involvement}

Patients and the public were not involved in any way in this study.

\section{Outcome measure}

The primary outcome measure was symptomatic thrombus of the limb in which the PICC was inserted, which included thrombus that occurred in the superficial venous system (SVT) or deep venous system (DVT) post PICC insertion. SVT was defined as occlusive thrombus in a superficial vein in which the PICC was inserted (basilic or cephalic veins). DVT included occlusive thrombus in the vein the PICC was inserted (if brachial) or if it extended into adjacent deep vasculature (axillary or subclavian veins). All cases were confirmed using ultrasound after clinical signs and symptoms triggered diagnostic testing while the PICC was still in situ or within 8 weeks of removal.

\section{Power analysis}

A power analysis using PASS V.11 (NCSS) determined that to achieve $80 \%$ power and 0.05 significance level, 2140 participants were required. A test of two independent proportions was based on an expected increased risk of thrombus (relative risk, $\mathrm{RR}=2.0$ ) where $80 \%$ have a CVR $\leq 45 \%$ and are considered low risk with a $3 \%$ thrombus rate and $20 \%$ a ratio of $\geq 46 \%$ will be high risk with a $6 \%$ thrombus rate. That is 1712 in the low-risk group and 428 in the high-risk group. These thrombus rates are based on previous research. ${ }^{15}$ It was possible for a patient to be in the study more than once (PICC reinsertion/exchange). However, we expected this to be a small proportion of participants and the impact of clustering to be minimal.

\section{Statistical analysis plan}

Descriptive statistics were used to present information about the study population. CVRs were determined by dividing PICC diameter (stated diameter or tapered diameter) by vein diameter and multiplying by 100 to generate a percentage. The association between the CVR and the risk of thrombus was analysed using a log binomial generalised linear model. This analysis was performed with all participants and according to diagnostic group. The same PICC design, with reverse taper capability was used in this study, but not all participants had the reverse taper portion inserted. As we were unsure about the accuracy of the PICC diameter (and hence CVR) of those with the tapered portion of the PICC inserted, analysis was repeated for those who didn't have the tapered portion of the PICC inserted. ROC analysis was used to plot the sensitivity and specificity of each ratio measurement using MedCalc for Windows, V.12.5 (MedCalc Software, Ostend, Belgium). The area under the curve (AUC) was used to identify the ideal CVR cut-off point with the aim to maximise sensitivity and specificity. All results with $\mathrm{p} \leq 0.05$ were considered statistically significant.

\section{RESULTS}

There were 2475 cases available, 37 were excluded due to missing data (11 with missing vein diameter and 26 missing diagnosis), leaving 2438 PICC insertions in the analysis. Nearly equal numbers of participants were male and female (table 2), with a mean age of 59 years old (SD 17.09). Most participants did not have a history of central venous access device (CVAD) insertion and had a cancer diagnosis. Participants with a cancer diagnosis had three times the risk of thrombosis than those with an infection as an underlying diagnosis. Those with a solid tumour appeared to have higher risk of thrombosis than those with a haematological malignancy, however, this was not statistically significant.

Most PICCs were inserted in the basilic vein in the right arm and required one needling attempt (table 3 ). Nearly equal numbers of single lumen (4Fr) and double lumen (5 Fr) PICCs were used. Most PICCs were verified using ECG, using a combination of securement devices and were inserted by staff with $3-5$ years of experience. The infusion of chemotherapy was associated with nearly four times the risk of thrombosis.

\section{Cases of thrombosis}

There were 39 cases of confirmed thrombosis, a rate of $1.6 \%$ (95\% CI $1.14 \%$ to $2.19 \%$ ). These comprised 13 cases of SVT (33\%), 5 cases of DVT (13\%) and 21 cases involving both the SVT and DVT (54\%).

\section{Catheter to vein ratio}

Based on ROC analysis, the CVR was not an effective diagnostic variable when treated as a continuous variable. The AUC was close to 0.5 when the ROC analysis was performed using the entire sample and according to diagnostic group. As the models lacked diagnostic ability, we analysed the association between risk of thrombosis and CVR cut-offs commonly used in clinical practice.

\section{All participants}

As per table 4, a CVR cut-off of $33 \%$ did not appear to be associated with risk of thrombosis, whereas, a $45 \%$ cut-off ( $\leq 45 \%$ vs $\geq 46 \%$ ) was predictive, with those with a higher ratio having more than twice the risk of thrombus ( $R R$ 2.30; $95 \%$ CI 1.202 to $4.383 ; \mathrm{p}=0.01$ ).

The analysis was repeated with participants who didn't have the tapered portion of the PICC inserted, that is, PICCs with an external length $\leq 6 \mathrm{~cm}$ were excluded (table 5). This comprised 1098 cases or $45 \%$ of the sample. Use of a CVR greater than $45 \%$ remained associated with more than twice the risk of thrombosis. When a 33\% CVR cut-off was analysed, the use of a CVR higher than 34\% appeared protective of thrombosis. However, neither of these results were statistically significant. 
Table 2 Participant factors and risk of thrombosis in individuals with a PICC

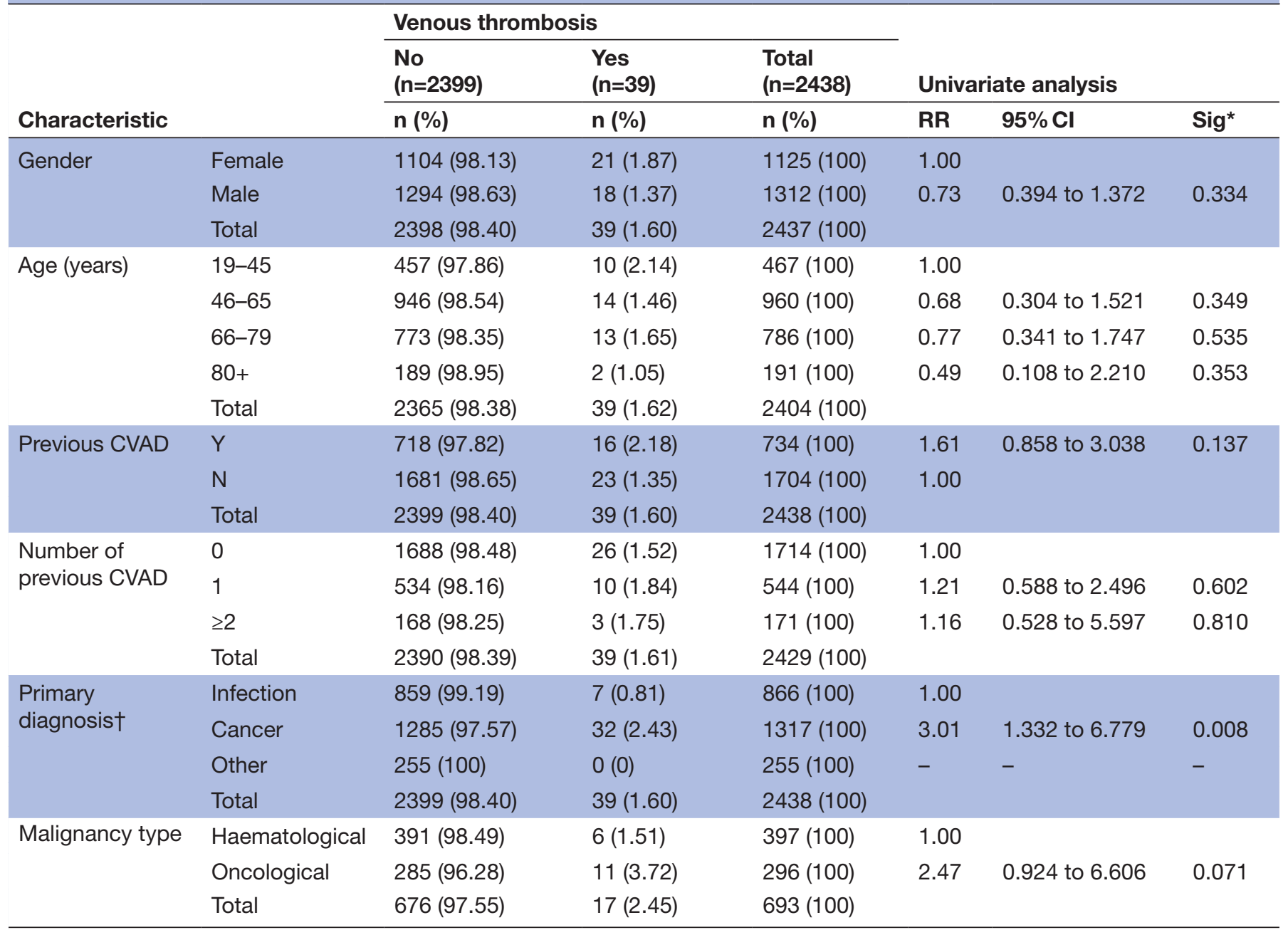

*Based on log binomial generalised linear model.

$\dagger$ As per treatment request.

CVAD, central venous access device; PICC, peripherally inserted central catheter; RR, relative risk.

\section{Effect of diagnosis on risk of thrombosis \\ Cancer}

When only participants with cancer were included in the analysis, a $45 \%$ CVR cut-off was associated with twice the risk of thrombosis (RR 2.10; 95\% CI 1.055 to 4.177; $\mathrm{p}=0.035$ ), while the use of a $33 \%$ CVR cut-off was not associated with risk (table 4). Although not statistically significant, when analysis was repeated with participants without the tapered portion of the PICC inserted, a CVR of $34 \%$ or greater appeared protective and the use of a CVR more than $45 \%$ was also associated with increased risk of thrombosis (table 5 ).

We then separated those with malignancy according to cancer type (693 participants had this information recorded) and repeated the analysis. For those with a haematological diagnosis, a CVR greater than 34\% was associated with slightly higher risk while a CVR greater than $45 \%$ was associated with more than three times the risk of thrombosis, although both results did not reach statistical significance. This analysis couldn't be repeated for those without the tapered portion of the PICC inserted in this group as there were no cases of thrombosis.

For those with a solid tumour, a CVR greater than $33 \%$ was associated with reduced risk of thrombosis and a CVR greater than $45 \%$ was associated with slightly elevated increased risk although the latter finding was not statistically significant. When the analysis was repeated for those who did not have the tapered portion of the PICC inserted, a CVR greater than $33 \%$ appeared protective, while a CVR higher than $45 \%$ was associated with more than four times increased risk, although the results did not reach statistical significance (table 5).

\section{Infection}

In participants with an infection or other non-cancer diagnosis, the association between a $45 \%$ cut-off and risk of thrombosis could not be analysed as none of the participants with a ratio of $46 \%$ and above developed thrombus (table 4). A 33\% CVR cut-off appeared to be protective in this cohort, with those with a CVR higher than $34 \%$ 


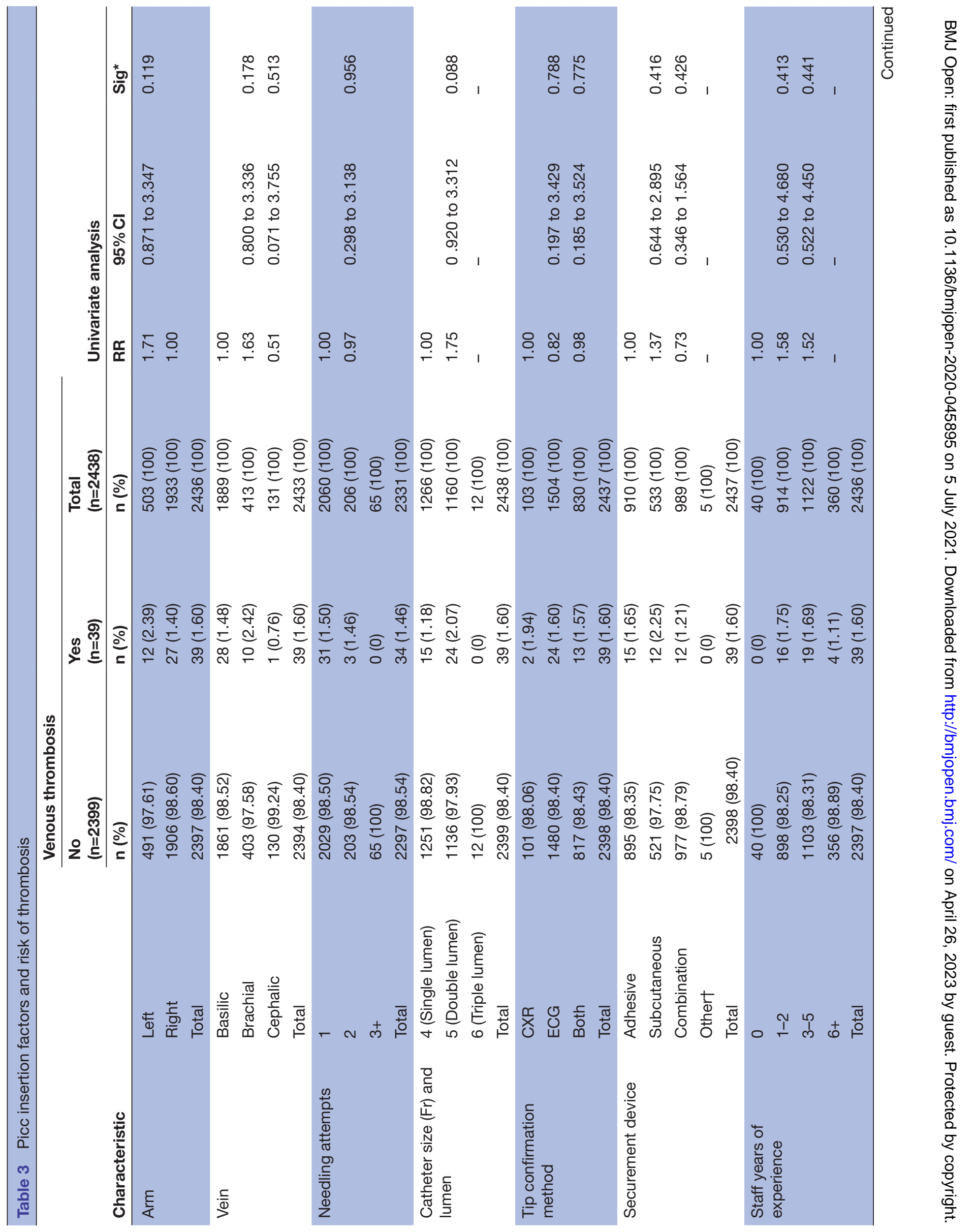




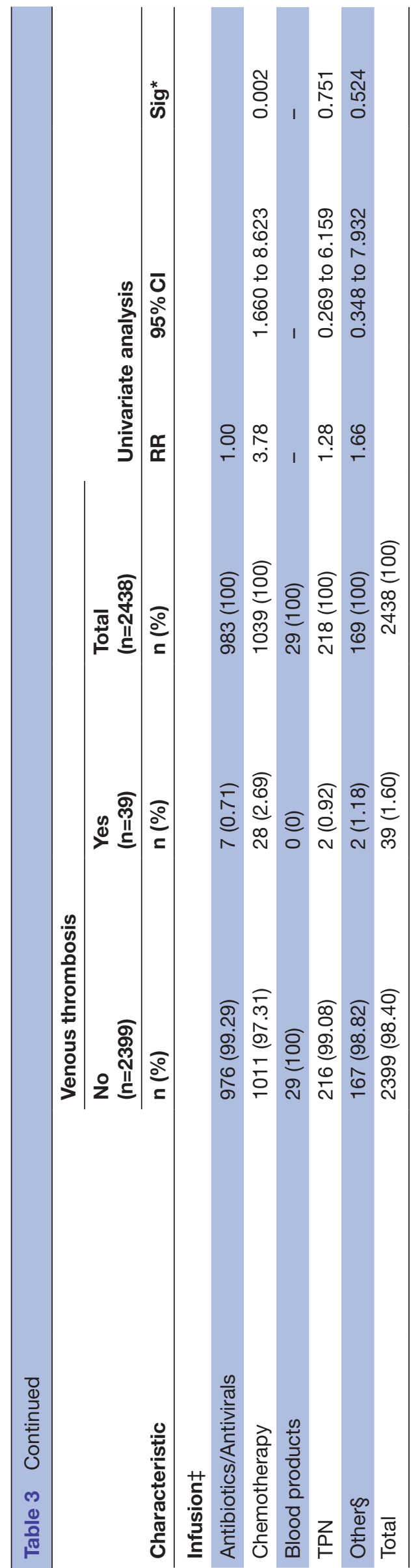

having less risk of thrombosis, although this was not statistically significant. Similar results were found when only non-tapered PICCs were included in the analysis (table 5).

\section{DISCUSSION}

We aimed to identify an optimal CVR cut-off for PICC insertion to prevent the risk of thrombosis. However, ROC analysis demonstrated that the CVR as a continuous measure was not an effective diagnostic variable overall, probably due to the low number of cases with higher CVRs. Hence, we analysed the risk of thrombosis associated with CVR cut-offs used in clinical practice.

A CVR greater than $45 \%$ was associated with twice the risk of PICC associated thrombosis when all participants were included in the analysis. When this analysis was performed according to diagnostic group, similar results were found in those with cancer. For those participants with malignancy, a CVR $45 \%$ cut-off was associated with more than twice the risk of thrombosis (RR 2.10; 95\% CI 1.055 to $4.177 ; p=0.035$ ). Although not statistically significant, this pattern continued when this was reanalysed with participants who did not have the reverse taper portion of the PICC inserted. Whereas the results for the 33\% CVR cut-off were not statistically significant in these cohorts.

Neither the $33 \%$ or $45 \%$ CVR cut-off produced statistically significant results in those with infection or other non-malignant conditions. We found a thrombosis rate of only $0.8 \%$ in this cohort and the analysis of thrombosis risk for each CVR was limited by a low number of cases of thrombosis. It is difficult to compare our results to previous research as most includes a mixed cohort (with consumers with cancer who have increased risk of thrombosis) and do not report results separately. Where research does examine only those receiving a PICC for the treatment of infection, with no underlying malignancy, insertion decisions such as the CVR are not documented. While the optimal CVR is not available for this cohort, we still recommend that a CVR limit is used until a more accurate estimate of risk is established.

The results from the present study suggest that the use of a CVR $\leq 45 \%$ is an important component in the strategies used during PICC insertion to reduce the risk of thrombosis in individuals with cancer requiring a PICC. ${ }^{3}$ Thrombosis is a significant adverse event in consumers with cancer and is associated with increased morbidity and mortality. ${ }^{16}{ }^{17}$ Factors such as the selection of an appropriate sized vein are especially important in the cancer patient cohort as larger, multilumen PICCs may be required which exacerbates thrombosis risk. These results support the use of a $45 \%$ CVR cut-off as advocated in the Infusion Nurses Society clinical guidelines (2021) and previous research that used ROC analysis to determine the optimal CVR cut-off. ${ }^{15}$

Our analysis of the risk associated with the CVRs used in clinical practice of those with haematological and oncological cancers separately should be interpreted with caution due to the low numbers of cases. However, it may 
Table 4 Catheter to vein ratio and risk of thrombosis according to diagnosis in individuals with a PICC

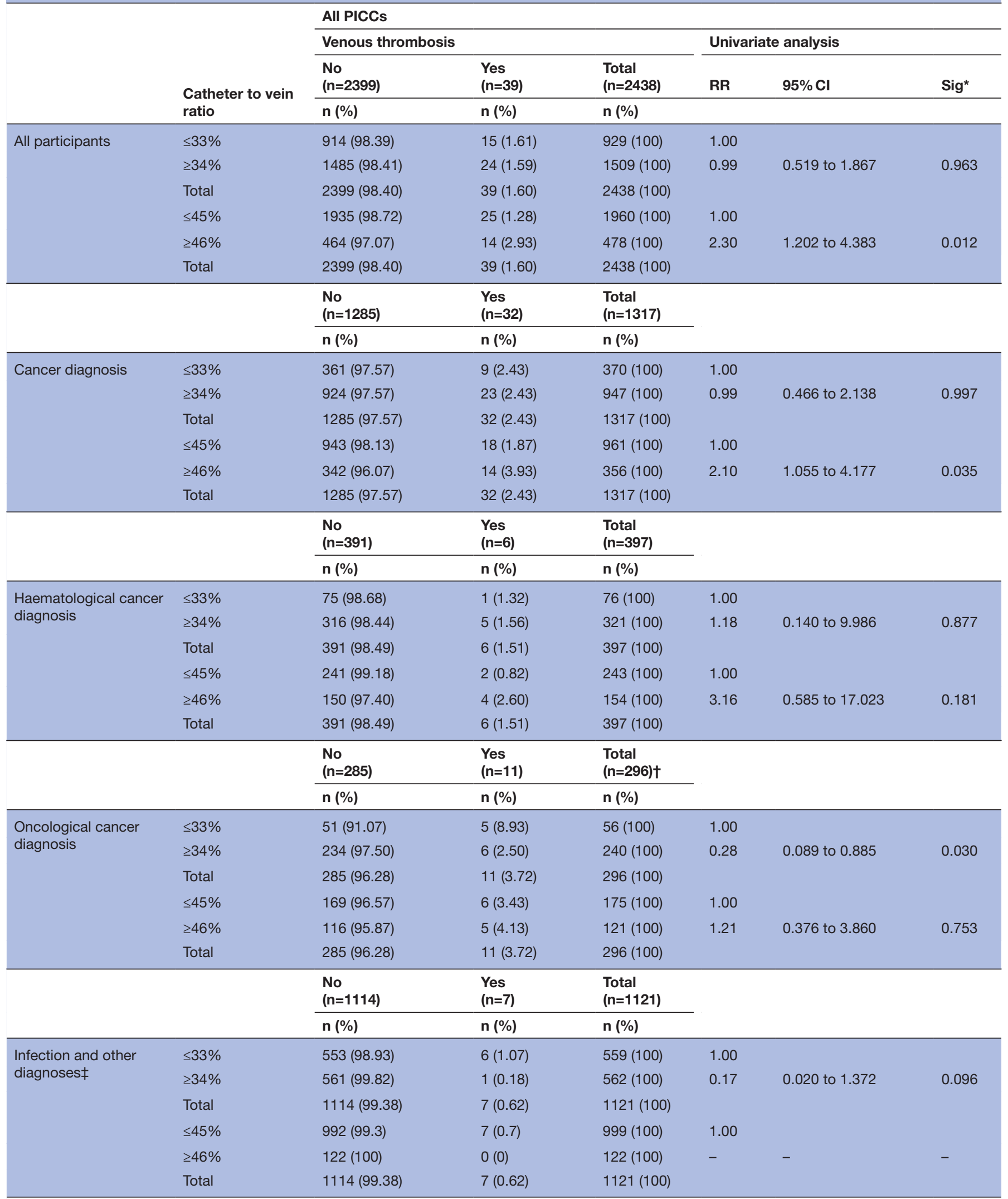

*Based on log binomial generalised linear mode.

tDoes not add up to total number due to missing data about cancer diagnosis.

‡Infection requiring intravenous antibiotics or other non-cancer diagnosis with difficult venous access requiring patient controlled analgesia, intravenous fluid, etc. PICC, peripherally inserted central catheter; RR, relative risk. 
Table 5 Catheter to vein ratio and risk of thrombosis in non-tapered PICCs

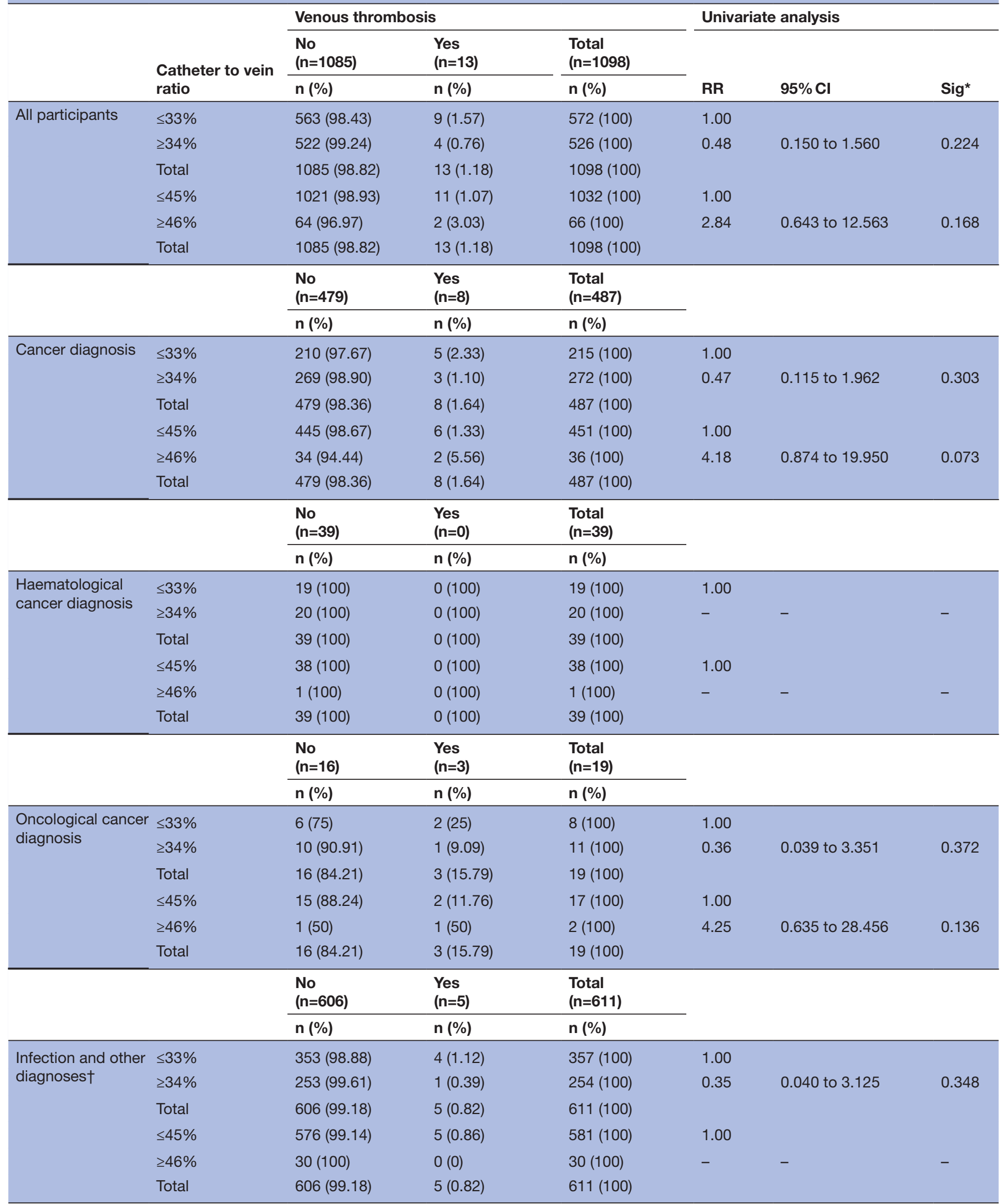

*Based on log binomial generalised linear model.

†Infection requiring intravenous antibiotics or other non-cancer diagnosis with difficult venous access requiring patient controlled analgesia, intravenous fluid, etc; one participant had missing external length.

PICC, peripherally inserted central catheter; RR, relative risk. 


\begin{tabular}{|c|c|}
\hline PICC size (Fr) & Minimum vein size \\
\hline $4 \mathrm{Fr}$ & $2.96 \mathrm{~mm}$ \\
\hline $5 \mathrm{Fr}$ & $3.70 \mathrm{~mm}$ \\
\hline $6 \mathrm{Fr}$ & $4.44 \mathrm{~mm}$ \\
\hline
\end{tabular}

CVR, catheter to vein ratio; PICC, peripherally inserted central catheter.

be that cancer type may influence risk. Previous research has found that those with a haematological cancer (Hodgkin lymphoma) experienced higher rates of PICC associated thrombosis than those with solid tumours. ${ }^{18}$ Further research is required to determine PICC associated thrombosis risk according to cancer type generally and may consider investigating risk according to specific diagnosis. A more nuanced understanding of the risk of PICC associated thrombosis for individual consumers would allow clinicians to provided targeted interventions for those most at risk.

\section{Implications for clinical practice}

This study indicates that the CVR should not exceed $45 \%$ for those with a cancer diagnosis. While the optimal CVR for those with infection and other non-malignant conditions is inconclusive, the low thrombosis rate found in the present study supports the use of minimum vein size strategy for all individuals requiring a PICC. The minimum vein diameters needed to achieve $\leq 45 \%$ CVR are detailed in table 6 . Many health consumers requiring a PICC will have a vein large enough for clinicians to adhere to these recommendations (we found that $80 \%$ of participants had a CVR $\leq 45 \%$, which demonstrates that adherence to the INS recommendations is feasible in most cases).

However, some health consumers will require a larger multi-lumen device and may not have an appropriate vein to accommodate the larger catheter. This is problematic, especially in those with cancer who are at higher risk and the use of thromboprophylaxis may be considered. Some evidence suggests that thromboprophylaxis reduces the risk of symptomatic CVAD associated thrombosis in individuals with cancer. A Cochrane review, ${ }^{19}$ found that thromboprophylaxis (low-molecular-weight heparin) halved the risk of thrombosis for those with a CVAD (RR $0.43,95 \%$ CI 0.22 to 0.81 ). This meta-analysis was composed of RCTs that included individuals with mostly solid tumours. Further research is needed in those with haematological malignancies. The use of thromboprophylaxis in individuals with haematological cancers also needs to be weighed against bleeding risk. ${ }^{20}$ Yet, thromboprophylaxis is used in some haematological cancer groups, for example, consumers with multiple myeloma taking thalidomide. ${ }^{21}$ An alternative vascular access device may also be considered for those most at risk. Research in individuals with cancer that has compared thrombosis risk according to different CVADs has found that PICCs were associated with more than seven times the risk of thrombosis (HR 7.48, 95\% CI 1.03 to 54.1, p=0.046) when compared with other non-tunnelled vascular access devices (central venous catheters), while implanted ports were associated with half the risk (HR $0.4795 \%$ CI 0.03 to $7.90 \mathrm{p}=0.597) .{ }^{22}$ Although insertion decisions such as the CVR are not documented in this research, perhaps large CVRs account for increased risk of thrombosis for those with a PICC.

\section{CVR with a tapered PICC}

Our results indicate that clinicians who use a reverse taper PICC should be aware of the increased diameter of the taper and depending on vein size, increased risk of thrombosis should the taper be advanced into the vein. It is important to recognise the significant impact that the taper has on PICC diameter. For example, a $6 \mathrm{Fr}$ longer tapered PICG would be $8 \mathrm{Fr}$ at the hub or $2.67 \mathrm{~mm}$ so to meet the $45 \%$ CVR cut-off, a vein would need to be $5.8 \mathrm{~mm}$ in diameter rather than $4.5 \mathrm{~mm}$ if it was inserted to the hub. To improve the accuracy of the PICC diameter for tapered PICCs when determining the CVR in clinical practice, clinicians could use the external length to determine the additional taper diameter as detailed in this study.

Alternatively, clinicians may avoid the use of the tapered part of the PICC by avoiding insertion of the taper. This will leave an external length of $\geq 7 \mathrm{~cm}$. While increased external length may be thought to increase dislodgement rates, anecdotally, this has not been the case with clinicians in this study. Some sites have introduced a subcutaneous device to fix the PICC in place which provides additional security for those with longer external lengths.

\section{Limitations}

A limitation with this study was the inclusion of PICCs with reverse taper design and resulting imprecise diameter to inform the CVR. While we developed an equation to determine the adjusted PICC diameter based on the external length this did not allow for the impact of subcutaneous tissue on this measurement. However, we expected this to have minimal impact on the overall CVR. Furthermore, we also presented analysis which only included non-tapered PICCs. There is a possibility that some participants presented to a regional hospital rather than the major hospitals in this study with symptoms of thrombosis, hence, we would miss cases of thrombosis. However, we expected this to be unlikely as most would be managed by their treating team at the specialist centres in the hospitals where the study was conducted. A further limitation, as with all retrospective studies is the reliance on existing data which in this study was evident in problems with missing data. For example, although participants were allowed into the dataset more than once, missing data meant that clustering could not be allowed for. However, we expected this to be a small proportion of participants and the impact of clustering to be minimal. 


\section{CONCLUSION}

This large study with over 2000 PICC insertions found a low rate of thrombosis which supports the use of this device to provide treatment for individuals with cancer and infection. The use of an appropriate vein size for PICC insertion is an important strategy to reduce PICC associated thrombosis in clinical practice. A CVR cut-off of $33 \%$ was not useful in predicting PICC-associated thrombosis in participants with cancer or other diagnoses. Our findings suggest that, in individuals with cancer, the CVR should not exceed $45 \%$, although further prospective studies are required to make definitive conclusions. This cut-off was not associated with risk of thrombosis for those with an infection and other non-cancer diagnosis. While further research is needed to determine the optimal CVR for those with infection, it is still recommended that the CVR is limited to reduce the risk of thrombosis.

\section{Author affiliations}

${ }^{1}$ Clinical and Health Sciences/Rosemary Bryant A0 Research Centre, University of South Australia, Adelaide, South Australia, Australia

${ }^{2}$ School of Nursing and Midwifery, National University of Ireland Galway, Galway, Ireland

${ }^{3}$ Alliance for Vascular Access Teaching and Research (AVATAR), Griffith University, Nathan, Queensland, Australia

${ }^{4}$ Clinical \& Health Sciences, University of South Australia, Adelaide, South Australia, Australia

${ }^{5}$ Vascular Access Team, Calvary Mater Hospital, Newcastle, New South Wales, Australia

${ }^{6}$ Peri-Operative Services, St Vincent's Hospital Sydney, Sydney, New South Wales, Australia

${ }^{7}$ Cancer Services, St George Hospital, Sydney, New South Wales, Australia

${ }^{8}$ Department of Anaesthesia and Pain Management, Capital and Coast District

Health Board, Wellington, New Zealand

${ }^{9}$ Medical Imaging, Fiona Stanley Hospital, Perth, Western Australia, Australia

Contributors RS: contributions to conception and design, literature search, data analysis and interpretation, writing, final approval of the version to be published. PC and GJ: contributions to conception and design, data interpretation, writing, final approval of the version to be published. AS, MY, TF, CK: contributions to conception and design, acquisition of data, data interpretation, writing, final approval of the version to be published. $A E, J C$ : contributions to conception and design, data analysis and interpretation, writing, final approval of the version to be published.

Funding This work was supported by a Pathfinder grant from the University of South Australia, Adelaide, Australia (School of Nursing and Midwifery). Award/grant number is not applicable. There was no conflict of interest, activities or potential for influencing this work by the funders.

Disclaimer The grant organisation had no financial interest or role in the design, conduct, analysis or manuscript preparation for this project.

\section{Competing interests None declared.}

Patient and public involvement Patients and/or the public were not involved in the design, or conduct, or reporting, or dissemination plans of this research.

\section{Patient consent for publication Not required.}

Ethics approval Ethics approval was obtained from the South Eastern Sydney Local Health District, Australia (HREC/17/POWH/174), Northern A Health and Disability Ethics Committee, New Zealand (17/NTA/264) and the University of South Australia (20026) Human Research Ethics Committees.

Provenance and peer review Not commissioned; externally peer reviewed.

Data availability statement Data are available on reasonable request from the corresponding author.

Open access This is an open access article distributed in accordance with the Creative Commons Attribution Non Commercial (CC BY-NC 4.0) license, which permits others to distribute, remix, adapt, build upon this work non-commercially, and license their derivative works on different terms, provided the original work is properly cited, appropriate credit is given, any changes made indicated, and the use is non-commercial. See: http://creativecommons.org/licenses/by-nc/4.0/.

ORCID iD

Rebecca Sharp http://orcid.org/0000-0003-3334-2990

\section{REFERENCES}

1 Rajasekhar A, Streiff MB. How I treat central venous access device-related upper extremity deep vein thrombosis. Blood 2017;129:2727-36.

2 Hughes ME. PICC-related thrombosis: pathophysiology, incidence, morbidity and the effect of ultrasound-guided placement technique on occurrence in cancer patients. J Assoc Vasc Access 2011:16:8-18.

3 Balsorano P, Virgili G, Villa G, et al. Peripherally inserted central catheter-related thrombosis rate in modern vascular access erawhen insertion technique matters: a systematic review and metaanalysis. J Vasc Access 2020;21:45-54.

4 Suleyman G, Kenney R, Zervos MJ, et al. Safety and efficacy of outpatient parenteral antibiotic therapy in an academic infectious disease clinic. J Clin Pharm Ther 2017;42:39-43.

5 Bertoglio S, Faccini B, Lalli L, et al. Peripherally inserted central catheters (PICCs) in cancer patients under chemotherapy: a prospective study on the incidence of complications and overall failures. J Surg Oncol 2016;113:708-14.

6 Jones D, Wismayer K, Bozas G, et al. The risk of venous thromboembolism associated with peripherally inserted central catheters in ambulant cancer patients. Thromb J 2017;15:25.

7 Kang J, Chen W, Sun W, et al. Peripherally inserted central catheterrelated complications in cancer patients: a prospective study of over 50,000 catheter days. J Vasc Access 2017;18:153-7.

8 Nifong TP, McDevitt TJ. The effect of catheter to vein ratio on blood flow rates in a simulated model of peripherally inserted central venous catheters. Chest 2011;140:48-53.

9 Chopra V, Kuhn L, Ratz D, et al. Vascular nursing experience, practice knowledge, and beliefs: results from the Michigan PICC1 survey. J Hosp Med 2016;11:269-75.

10 Evans RS, Sharp JH, Linford LH, et al. Reduction of peripherally inserted central catheter-associated DVT. Chest 2013;143:627-33.

11 Pittiruti M, Emoli A, Porta $\mathrm{P}$, et al. A prospective, randomized comparison of three different types of valved and nonvalved peripherally inserted central catheters. J Vasc Access 2014;15:519-23.

12 Walters B, Price C. Quality improvement initiative reduces the occurrence of complications in peripherally inserted central catheters. J Infus Nurs 2019;42:29-36.

13 Cotogni P, Barbero C, Garrino C, et al. Peripherally inserted central catheters in non-hospitalized cancer patients: 5-year results of a prospective study. Support Care Cancer 2015;23:403-9.

14 Gorski LA, Hadaway L, Hagle ME, et al. Infusion therapy standards of practice, 8th edition. J Infus Nurs 2021;44:S1-224.

15 Sharp R, Cummings M, Fielder A, et al. The catheter to vein ratio and rates of symptomatic venous thromboembolism in patients with a peripherally inserted central catheter (PICC): a prospective cohort study. Int J Nurs Stud 2015;52:677-85.

16 Blom JW, Doggen CJM, Osanto S, et al. Malignancies, prothrombotic mutations, and the risk of venous thrombosis. JAMA 2005;293:715-22.

17 Sørensen HT, Mellemkjaer L, Olsen JH, et al. Prognosis of cancers associated with venous thromboembolism. $N$ Engl J Med 2000;343:1846-50.

18 Ellis M, Okano S, McCann A, et al. Catheter-Related thrombosis incidence and risk factors in adult cancer patients with central venous access devices. Am Soc Hematology 2017;50:1475-82

19 Kahale LA, Hakoum MB, Tsolakian IG, et al. Anticoagulation for the long-term treatment of venous thromboembolism in people with cancer. Cochrane Database Syst Rev 2018;6:CD006650.

20 Niers TMH, Di Nisio M, Klerk CPW, et al. Prevention of catheterrelated venous thrombosis with nadroparin in patients receiving chemotherapy for hematologic malignancies: a randomized, placebo-controlled study. J Thromb Haemost 2007;5:1878-82.

21 Geerts WH, Bergqvist D, Pineo GF, et al. Prevention of venous thromboembolism: American College of chest physicians evidence-based clinical practice guidelines (8th edition). Chest 2008;133:381S-453

22 Ellis M, Okano S, McCann A. Catheter-Related thrombosis incidence and risk factors in adult cancer patients with central venous access devices. Blood 2017;130:2096. 